American J. of Engineering and Applied Sciences 3 (1): 159-170, 2010

ISSN 1941-7020

(C) 2010 Science Publications

\title{
Simulation of Airflow and Aerodynamic Forces Acting on a Rotating Turbine Ventilator
}

\author{
A.S. Farahani, N.M. Adam and M.K.A. Ariffin \\ Department of Mechanical and Manufacturing Engineering, University Putra Malaysia, \\ 43400 Serdang, Selangor, Malaysia
}

\begin{abstract}
Problem statement: Rotating turbine ventilators were generally found in most countries. They were simple in structure, light in weight and cheap to install. It was quite surprising that, the aerodynamics of this common device had not been numerically examined and the design process of most of these ventilators had developed progressively through trial and error methods. Approach: This study was concerned with performing simulation of airflow using CFD technique code name FLUENT so as to visualize the flow behavior around and within a rotating turbine ventilator in addition to determining the aerodynamic forces acting on this device during its operation. To achieve that, the realizable k- $\varepsilon$ and RSM turbulence models were used by taking advantage of moving mesh method to simulate the rotation of turbine ventilator and the consequent results were obtained through the sequential process which ensured accuracy of the computations. Results: The results confirmed that, the realizable $k-\varepsilon$ model can exhibit a reasonable performance, however not as competence as the RSM model, but of much less computation time. Conclusion/Recommendations: Results from this study, besides ensuring the reliability of utilizing the CFD method in design process of future turbine ventilators, would lead us to a conspicuous progress on increasing the efficiency at reduced cost of wind driven ventilators and similar devices.
\end{abstract}

Key words: CFD, moving mesh, turbine ventilator

\section{INTRODUCTION}

Many Southeast Asian countries have experienced high economic growth accompanied by rapid urbanization over the last few decades. This resulted in tremendous increase in energy consumption especially in urban areas. Since most of the cities in this region experience hot-humid climate all the year round, it is particularly important to develop passive cooling techniques in order to reduce energy demand caused by the growing use of air-conditioners. Natural form of ventilation is one of the low-cost passive cooling techniques that may contribute to reducing the cooling load of buildings and to improving thermal comfort of occupants (Kubota et al., 2009).

Natural ventilation uses the natural forces of wind pressure and stack effects to redirect the movement of air through dwellings. Various natural ventilation techniques are used in low energy building design and they are classified as: Passive wind driven ventilation, directed passive wind driven ventilation and active wind driven ventilation.

In passive wind driven ventilation category, devices and methods are passive in nature and primarily use wind-induced effects as motive forces for providing ventilation. Some examples of these devices and methods are; window openings, atria and courtyards. In directed passive wind driven ventilation technique, devices take advantage of the partial negative pressure created when winds blow across openings, such as wind cowls/scoops. Finally, in active wind driven ventilation group, ventilators are operating by the action of centrifugal force by creating a pressure difference, which helps drive out the air from inside an enclosed space (Khan et al., 2008).

In the present study, a ventilator from the last group namely, turbine ventilator, is numerically examined. A turbine ventilator is a wind-driven air extractor. It includes a number of vertical vanes (curved or straight blades) in a spherical or cylindrical array mounted on a frame. When wind blows on the aerofoil vanes the resultant lift and drag forces cause the turbine to rotate. This rotation produces a negative pressure inside the turbine which extracts air. Air enters the turbine axially via the base duct and is then expelled radially. In the absence of wind, a turbine ventilator facilitates ventilation using stack effects (Rashid and Ahmed, 2003).

Corresponding Author: A.S. Farahani, Department of Mechanical and Manufacturing Engineering, University Putra Malaysia, 43400 Serdang, Selangor, Malaysia 
A turbine ventilator concept was originally patented as early as in 1929 by Meadows (1929), who described it as a rotary ventilator. A number of scientists have tested the turbine ventilator experimentally in the state of some features such as the effects of this device in ventilation rate, the effect of size and shape of blades on its performance and the temperature difference caused by the turbine ventilator (Lai, 2003; Revel, 1998; West, 2001; Wilson, 1994; Dale and Ackerman, 1993; Lai and Kuo, 2005). In other group of studies, however, the performance of turbine ventilator has been examined by redesigning the blades shape, combining a DC fan with turbine ventilator and considering the turbine ventilator as a combination of backward curved centrifugal fan and wind turbine (Marchman, 1982; Havens, 2004; Lai, 2006). In last group of investigations, pressure distributions around and through turbine ventilator have been measured besides determining the aerodynamic forces to analyze the flow field and aerodynamic performance of a rotating and static turbine ventilator experimentally (Rashid and Ahmed, 2003; Pisasale, 2004). The distinct lack of literature on these ventilators has inspired this investigation. In the present study, numerical simulations of flow field around and through a verticalvane turbine ventilator have been performed for the purpose of visualizing the continuous pressure distributions to complete previous studies besides comparing the simulated flow field and aerodynamic forces with experimental data to validate the numerical model used.

For this study, the commercial CFD code of choice was Fluent 6.3. Modeling of the prototype has been made by using Solid-works 2007 and Gambit 2.0 software was used for generating the appropriate mesh. All the simulations were performed in a threedimensional space domain. The turbulence model is of particular importance in this application due to complexity of the airflow through and around the turbine ventilator. The Standard k- $\varepsilon$ model, which is the most common method applied in the majority of CFD applications, cannot accurately simulate the flow separation region that occurs at the tips of the rotating blades. This can lead to significant errors in the overall analysis (Cochran, 2004). Therefore, the Realizable k- $\varepsilon$ model which satisfies certain mathematical constraints on the Reynolds stresses, consistent with the physics of the turbulent flows and RSM model, which provides a physically realistic and accurate prediction of the flow field for computation of turbulent flow in turbine rotor blades, are chosen to be the appropriate models of choice for this study (Lakshminarayana, 1996).

\section{MATERIALS AND METHODS}

The numerical method utilized for the simulation had a pressure based solver with implicit formulation. To compute the secondary diffusion terms and velocity derivatives the green-gauss node based method was used to discretize the convection and diffusion terms in the flow conservation equations. A secondary interpolation method with a high reliability level has been employed and the method of choice for the pressure-velocity coupling was PISO algorithm. Finally, the standard scheme was used as the pressure interpolation scheme and density, momentum and turbulent kinetic energy were set to second order upwind scheme.

Geometry and boundary conditions: In the initial phase the major solid and fluid region interfaces are established. The dimensions of the prototype model are given in Fig. 1.

The coordinate system origin used is located at the center of the model, with $\mathrm{x}$ ordinate in the free stream direction and the $\mathrm{y}$ ordinate pointed up as the $\mathrm{x}, \mathrm{y}$ plane and the $\mathrm{xz}$ plane corresponding respectively to the longitudinal and vertical sections of symmetry. Also, it must be noted that the ventilator is rotating about the $y-$ axis on the xz-plane.

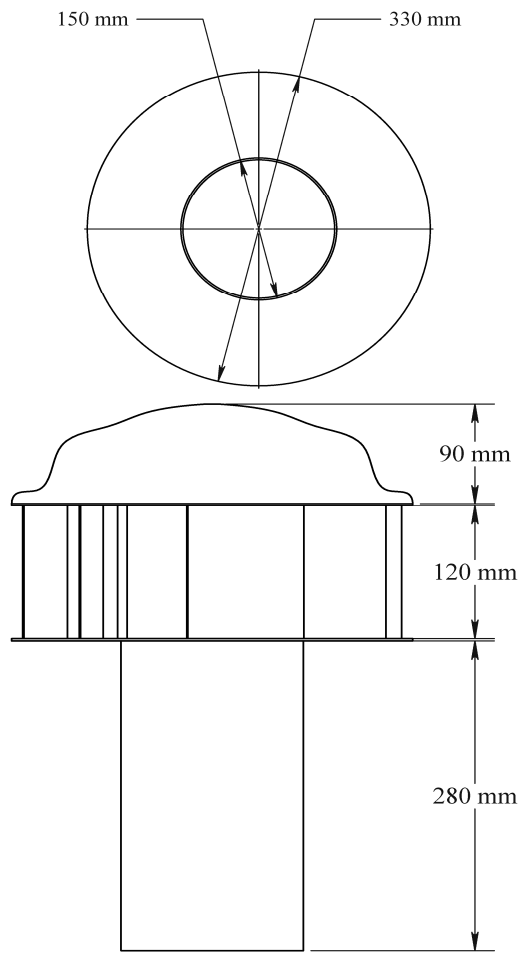

Fig. 1: Dimensions of the turbine ventilator, bottom and front view, respectively 


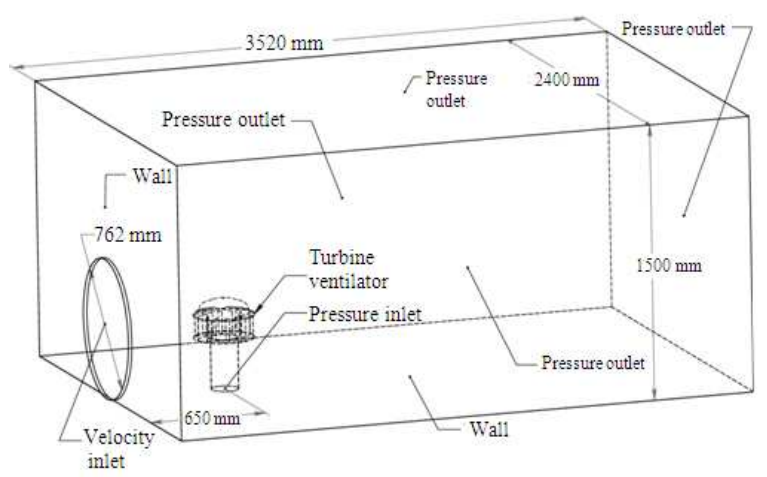

Fig. 2: Control volume and specified domains

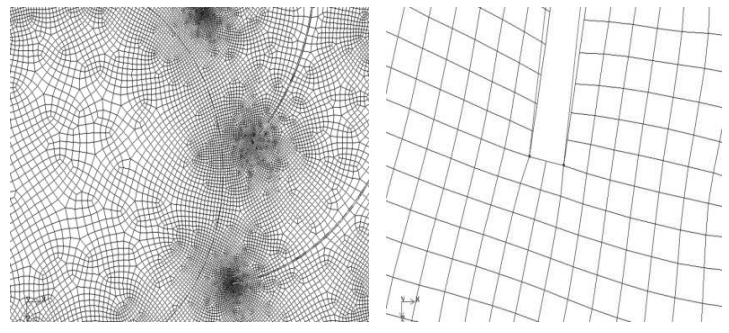

Fig. 3: Boundary layer mesh near the leading edge of turbine ventilator blade in wall function method

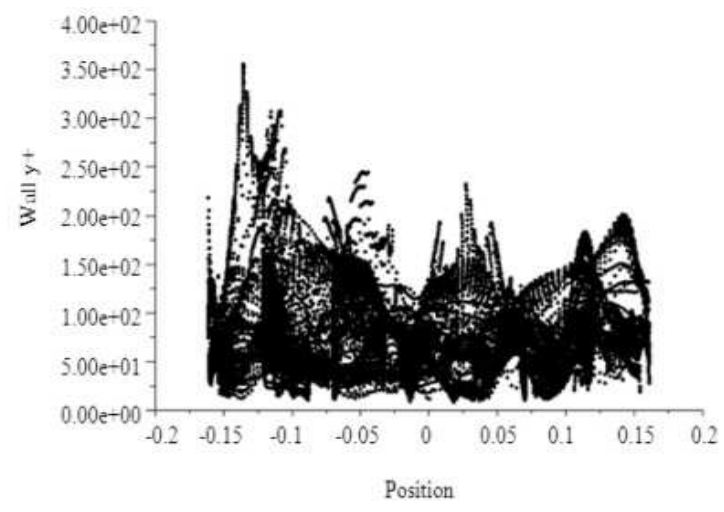

Fig. 4: Wall $\mathrm{y}^{+}$, obtained from simulation of turbine ventilator using wall function method

The model is vertically centered in a cubic computational domain with the height of $4.5 \mathrm{~d}$ and width of $7.2 \mathrm{~d}$, where $\mathrm{d}$ is the rotor diameter. The distance from the model surfaces to the inflow and outflow planes must be long enough to avoid the influence of the model on the total pressure there, besides, verifying the usual computational requirements, these lengths must also be chosen to match the dimensions of the wind tunnel section. Thus, the computational domain is extended by $2 \mathrm{~d}$ upstream and $7.6 \mathrm{~d}$ downstream the body, as shown in Fig. 2.

The proper mesh for wall function treatment used in realizable $\mathrm{k}-\varepsilon$ and RSM models were generated using the Quad-Pave scheme. This scheme gives the possibility to adjust different cell height at the first row for one specific wall boundary which is essential for generating good mesh resolution on critical areas such as blade leading and trailing edges. Therefore, this scheme is used and adapted to obtain the desired $\mathrm{y}^{+}$ values of $11.225<\mathrm{y}^{+}<300$, needed for the accurate simulation of turbine ventilator, Fig. 3. The resultant $y^{+}$ values are shown in Fig. 4.

For all CFD simulation cases the room domain was dicretized in the range of 3.5 million cells. Grid independence results were established by changing the grid sizes and comparing the outcomes. Two selective cases at $v_{\mathrm{f}}=7$ and $\mathrm{v}_{\mathrm{f}}=20 \mathrm{~m} \mathrm{sec}^{-1}$ for Inlet, were simulated with a finer mesh of approximately 4 million cells. The difference between the average drag coefficient and lift coefficient were found to be 1 and $1.55 \%$, respectively. However it must be noted that, due to computer power limitation, the examinations of finer meshes were aborted. Therefore, the presented mesh independence tests seem to be enough accurate for the study of turbine ventilator.

Due to applicability and accuracy of sliding mesh method, this method is applied for the simulation of turbine ventilator rotation and periodic blade pitch variation. The appropriate angular velocity corresponding to respective free-stream velocity is used from wind tunnel test data (Pisasale, 2004) which, could be derived from $\Omega / \mathrm{v}_{\mathrm{f}}=3.4304$, where, $\Omega(\mathrm{rad}$ $\mathrm{sec}^{-1}$ ), is the angular velocity. The consequent Tip Speed Ratio is equal to 0.56 and is constant for entire range of velocity. For all the simulations, the flow was assumed to have $0.2 \%$ Turbulence Intensity (TI) at the inlet (Gatto et al., 2001).

In the study of turbine ventilator, a time-periodic solution is desired for simulation of rotating turbine ventilator characteristics where startup transient behavior is not of interest. Therefore, once this startup phase has passed, the flow will start to exhibit timeperiodic behavior. For rotation of turbine ventilator, the period (in seconds) can be calculated by dividing the sector angle of the domain (in radians) by the rotor speed (in radians $\left.\sec ^{-1}\right),(T=\theta / \Omega)$. When the solution field does not change from one period to the next (for example, if the change is less than 5\%), a time-periodic solution has been reached (Reference Manual, 2006). In this study the solution stability is judged by plotting a running average of the lift coefficient, as shown in Fig. 5. 


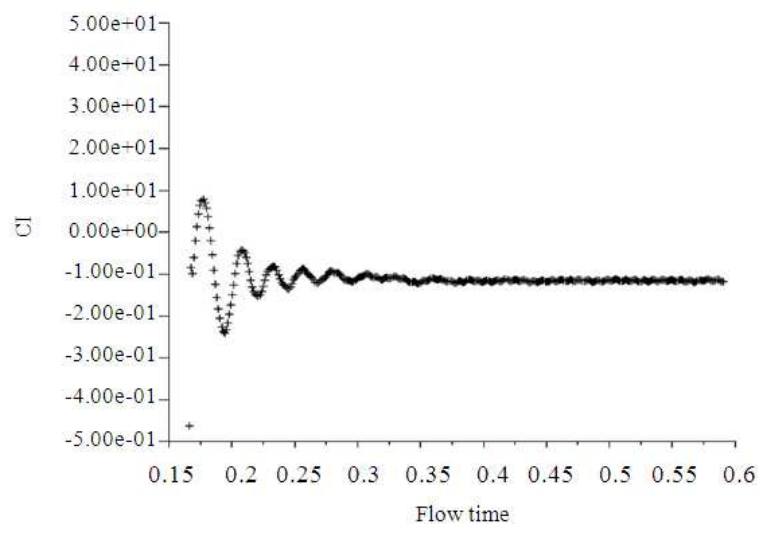

Fig. 5: Solution convergence: Turbine average lift coefficient

The residual convergence value used in this study was $10^{-4}$. Smaller residual value does not result in any noticeable change of the calculated results but required extensively more computation time. To achieve a solution which is independent of the time step size, it was found that the time step has to be small enough so that a single step does not rotate the interface a distance greater than the smallest cell size. As a result, the time step used was quite small, typically on the order of $0.001 \mathrm{sec}$. In most cases, three revolutions of the rotor are required to reach a stable solution. (Note, each revolution of the rotor requires $35-45 \mathrm{~h}$ of computational time on a 2.8 $\mathrm{GHz}$ Core 2 Duo,4 GB RAM workstation, depending upon the size of the mesh file).

\section{RESULTS}

Flow visualization results and discussion: For visualizing the flow field around and within the rotating turbine ventilator, two velocities of $\mathrm{v}_{\mathrm{f}}=7$ and $\mathrm{v}_{\mathrm{f}}=20 \mathrm{~m} \mathrm{sec}^{-1}$, according to experiment (Pisasale, 2004), were considered as the free-stream velocities. To validate the results, same cut-planes as experiment for plotting the pressure counters and velocity magnitudes were taken.

The final meshes shown in the Fig. 6-10 have the ordinates non-dimensionalized with the rotor diameter $\mathrm{d}_{1}$ and the new ordinates have the subscript $\mathrm{d}_{1}$ for instance $Y_{d}=Y / d$. Furthermore, the pressure has been non-dimensionalized by the free stream dynamic pressure and the same scale as experiment is used which allows direct comparison. It must be noted that, in the Fig. 6c-10c the mean flow dynamics have been represented by streamtraces, which like streamlines are tangent to the velocity vectors. However, unlike a streamline, velocity magnitude has been represented by the arrows on the streamtraces. These arrows are like particles in the flow, the greater the distance between particles on the same line, the greater the velocity magnitude in this flow region. Below, the results from two cut-planes are given and discussed.

At $Y_{d}=0.787$, the cut-plane is below the bottom of rotating ventilator top and the flow at $v_{\mathrm{f}}=7 \mathrm{~m} \mathrm{sec}^{-1}$ is considered first. The first notable feature in this cutplane is the incidence of flow by the rotor, which divides the front high pressure region into two regions. It can be seen from the pressure contours, Fig. $6 \mathrm{~b}$ and c, that these regions are separated by the circular arc low pressure strip which extends to right and joins the other low pressure strip diagonally parallel to the streamtraces. Although this low pressure strip is not clear in experiment result, it is obviously exhibited by velocity magnitudes from both turbulence models, Fig. 6a and b. This strip widens the wake behind the turbine ventilator to the right. Formation of these strips is due to the tangential velocity of the rotor which increases with increase in rotor radius.

Towards the cylindrical base, the pressure is fractionally higher, before a small low pressure region can be seen on the right surface of the base, slightly towards the back. A similar region is also found in the corresponding position on left side but of greater amount due to direction of ventilator rotation. Also, there can be seen two separate low pressure regions in the wake, Fig. 6c, which are due to vortices. Furthermore, the pressure contours behind these could also indicate a larger left vortex. The simulation results of Realizable k- $\varepsilon$ and RSM models, (Fig. 6a and b), on previously discussed regions are in good agreement with experiment.

The other notable feature in this cut-plane is the pressure distribution and the velocity magnitude presented by CFD results inside the cylindrical base. Unfortunately, there is no experimental result about the flow behavior inside the base. Thus, by checking the computation results, a low pressure region in the right side inside the base with a small induced clockwise circulation which must be due to the above pressure distribution inside the blade area caused by ventilator rotation is predicted.

Interestingly, the flow behavior at higher speed on the same cut-plane shown in Fig. 7, is very similar to the results at lower speed, Fig. 6. There is once again notable incidence which divides the front high pressure region. As well the two pressure regions are on the cylinder surface, although the left region is more obviously greater in size than the right, which is due to increase in free stream velocity and consequently the angular velocity of the rotor. 


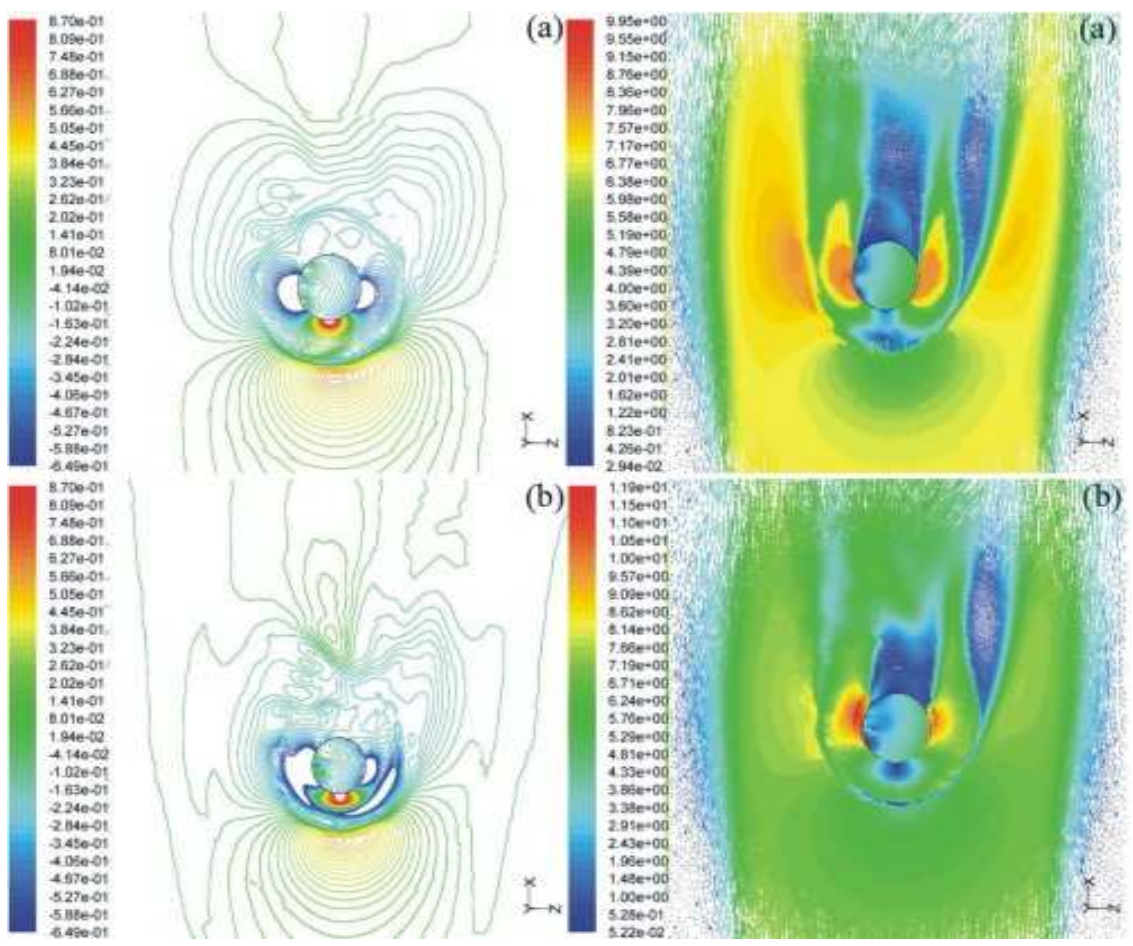

(c)
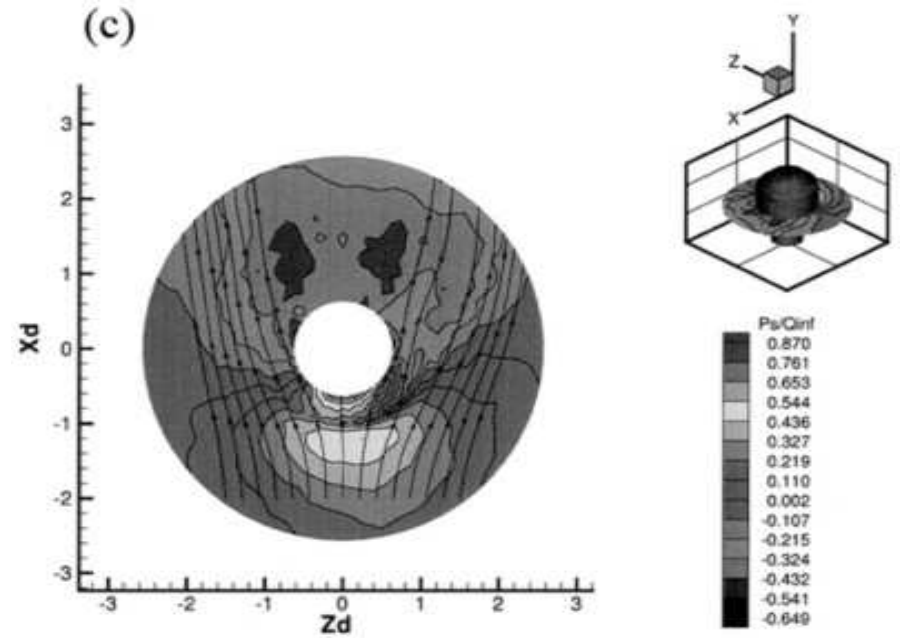

Fig. 6: Pressure contours and velocity magnitudes at $Y_{d}=0.787$ and $v_{f}=7 \mathrm{~m} \mathrm{sec}^{-1}$ for Realizable k- $\varepsilon$ (a), RSM (b) and experimental result (c), respectively

The two distinct pressure regions in the wake cannot however be seen in experiment, but as it is shown in computation results and the shape of the pressure contours further out indicate that they may be present, with the left being once again larger than the right.

At $Y_{d}=1.015$, the mid-plane through the rotor from experiment, Fig. 8c, it is difficult to recognize any structure in the pressure contours in this cut-plane.
There does however appear to be a wide region of low pressure extending to the right in the wake. There seems also to be a small low pressure region near the surface of the rotor to the left and in the wake. The streamtraces are tighter, particularly on the right side, comparing to previous cut-plane in Fig. 6c and so it appears that, due to the shape of pressure contours and the behavior of the streamtraces, the circulation has increased. 

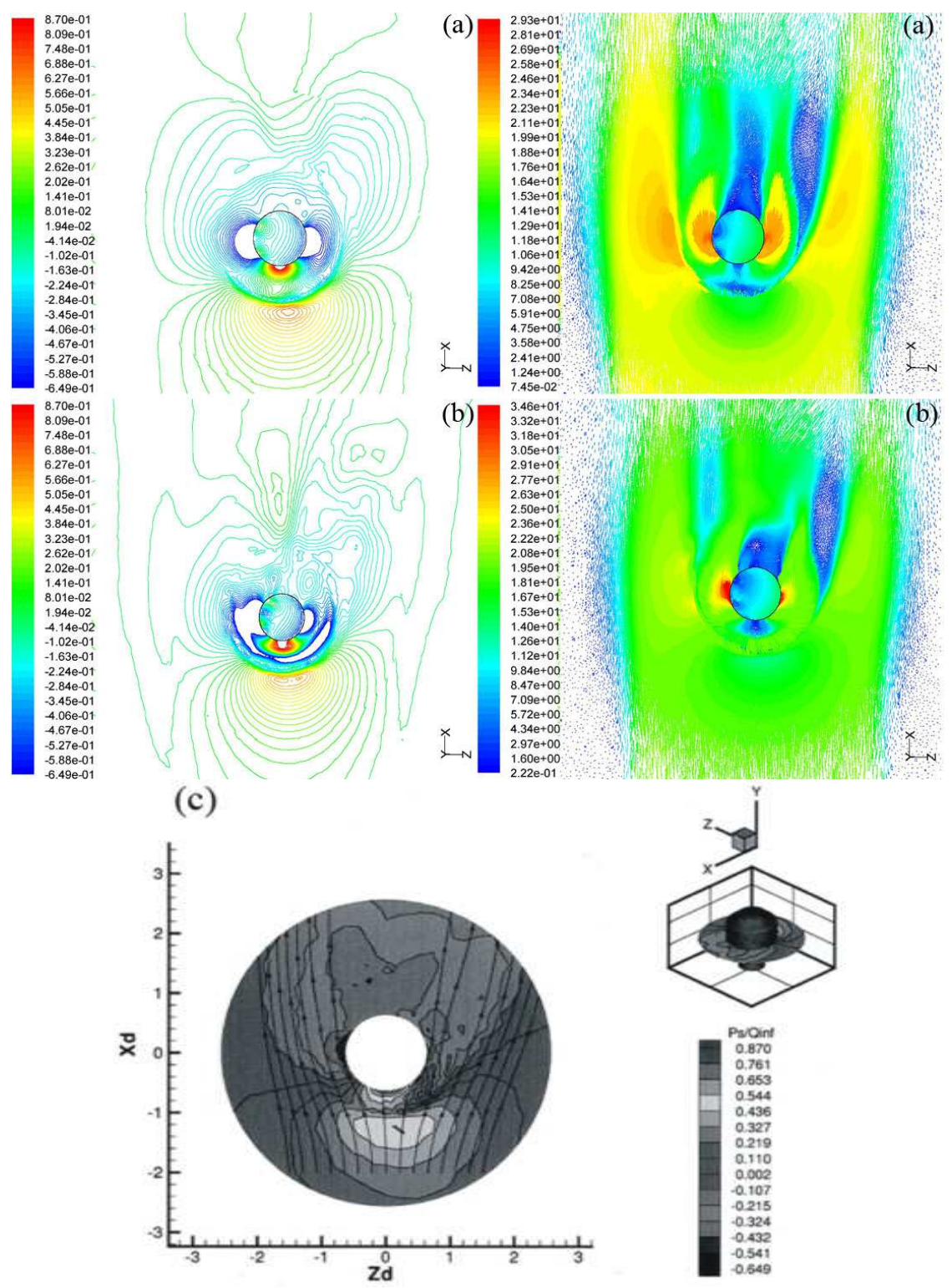

Fig. 7: Pressure contours and velocity magnitudes at $Y_{d}=0.787$ and $v_{f}=20 \mathrm{~m} \mathrm{sec}^{-1}$ for Realizable k$\varepsilon$ (a), RSM (b) and experimental result (c), respectively

By checking the simulation results shown in Fig. $8 \mathrm{a}$ and $\mathrm{b}$, it is indicated that, supporting the experiment observation, the wake has extended in width and is divided into two streams with the right one being larger than the left one. Now the effect of ventilator rotation is obviously illustrated and the small low pressure region on the left side of the wake observed from experiment is seem to be as a result of flow separation at the trailing edge of the blade. Both models are in good agreement with experiment however, the RSM model shows more competence than the other model in predicting the vortices and pressure distribution in this region.

One of the most motivating parts of this study is visualizing the flow behavior and pressure distribution through the rotating ventilator blades. The experimental flow visualization was done for the area inside the rotating rotor above the cylindrical base excluding the area between $b$ lades (Pisasale, 2004), in which has not experimentally examined yet. In that study three cut-planes are considered to visualize the flow field inside the rotating 

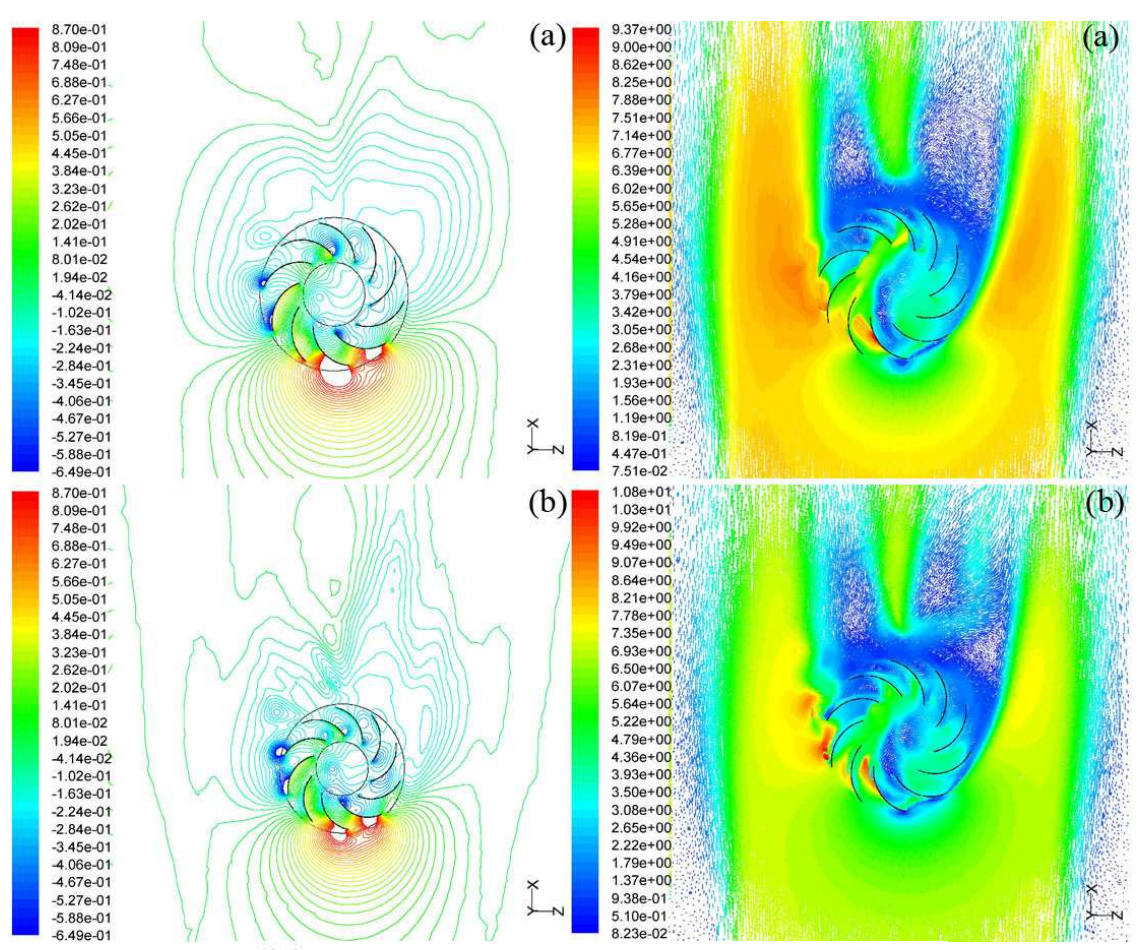

(b)

$1.08 \mathrm{e}+01$
$1.03 \mathrm{e}+01$

$7.48 \mathrm{e}-01$
$6.88 \mathrm{e}-01$
$6.27 \mathrm{e}-01$
$5.66 \mathrm{e}-01$
$5.05 \mathrm{e}-01$
$4.45 \mathrm{e}-01$
$3.84 \mathrm{e}-01$
$3.23 \mathrm{e}-01$
$2.62 \mathrm{e}-01$
$2.02 \mathrm{e}-01$
$1.41 \mathrm{e}-01$
$8.01 \mathrm{e}-02$
$1.94 \mathrm{e}-02$
$-4.14 \mathrm{e}-02$
$-1.02 \mathrm{e}-01$
$-1.63 \mathrm{e}-01$
$-2.24 \mathrm{e}-01$
$-2.84 \mathrm{e}-01$
$-3.4 \mathrm{e}-01$
$-.06 \mathrm{e}-01$
$-4.67 \mathrm{e}-01$
$-5.27 \mathrm{e}-01$
$-5.88 \mathrm{e}-01$
$-6.49 \mathrm{e}-01$

(c)
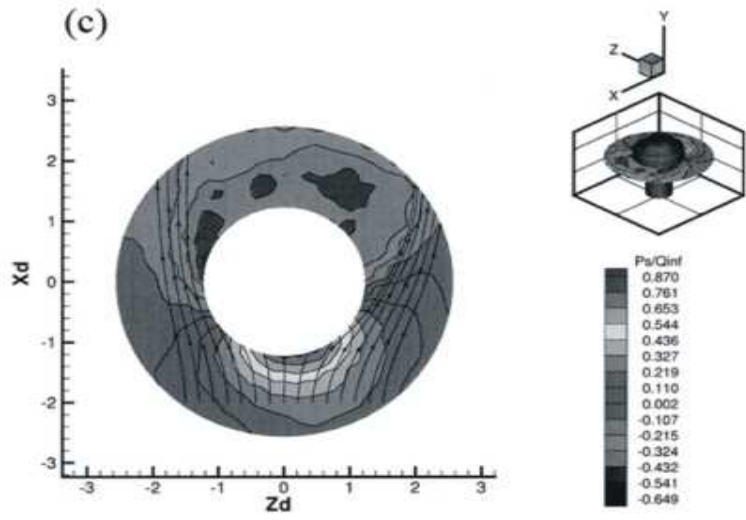

Fig. 8: Pressure contours and velocity magnitudes at $Y_{d}=1.015$ and $v_{f}=7 \mathrm{~m} \mathrm{sec}^{-1}$ for Realizable k- $\varepsilon$ (a), RSM (b) and experimental result (c), respectively

rotor at the free stream velocity of $v_{\mathrm{f}}=7 \mathrm{~m} \mathrm{sec}^{-1}$. These cut-planes are located at different positions compare to those were used for visualization of flow field around ventilator. Thus, a direct comparison is not possible to be made between simulation results and the mentioned experiment.

The experimental results are given in Fig. 9. Three vertical positions were selected to examine how the axial pressure gradient develops through the ventilator. These correspond to planes cut through the rotor at $1 / 2$, $1 / 4$ and 3/4 of the rotor axial blade height H. In Fig. 9 the free stream direction is down the page and the drawn circle represents the wall of the cylindrical base.
The $\mathrm{x}$ and $\mathrm{z}$ ordinates have been non-dimensionalized with the radius of the base:

$$
\mathrm{R}=\mathrm{D} / 2
$$

where, $\mathrm{D}=0.15 \mathrm{~m}$ and the rotation of the ventilator is clockwise.

Checking the simulation results at $Y_{d}=1.015$ and $\mathrm{v}_{\mathrm{f}}=7 \mathrm{~m} \mathrm{sec}^{-1}$ shown in Fig. 8a and b, there are high pressure regions where the flow enters the ventilator blades area due to incidence of flow with rotating blades. These high pressure regions give the ventilator its torque. 


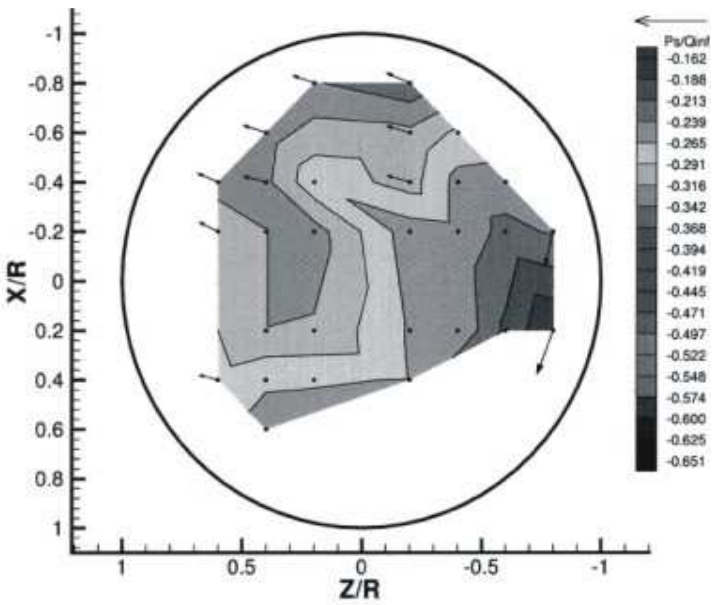

(a)

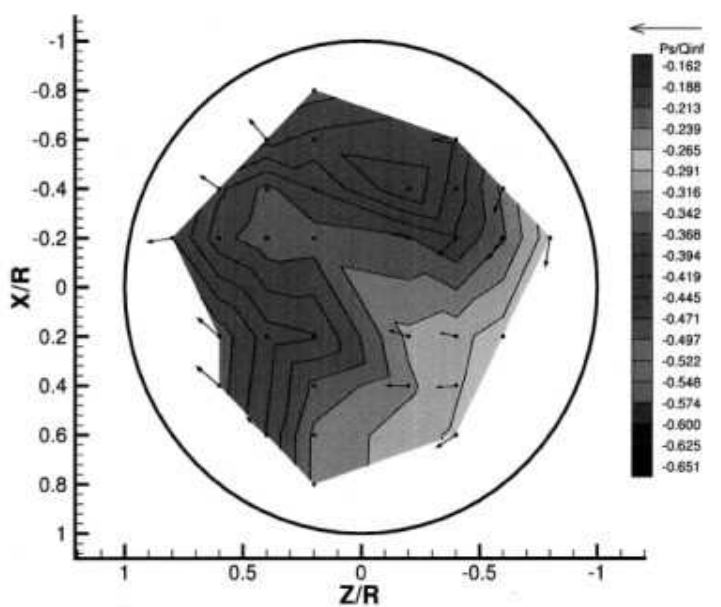

(b)

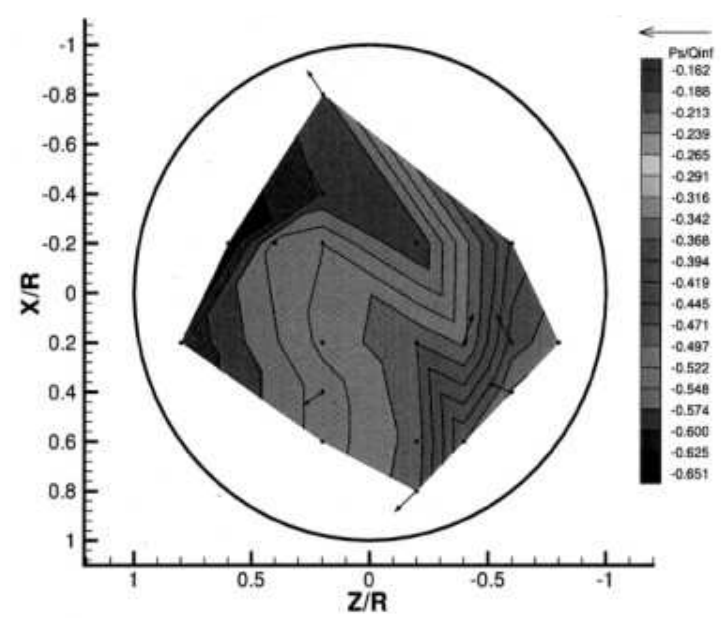

(c)

Fig. 9: The planner pressure distribution inside the ventilator at cut plane of (a) $\mathrm{Y} / \mathrm{H}=1 / 4$, (b) $\mathrm{Y} / \mathrm{H}=2 / 4$ and (c) $\mathrm{Y} / \mathrm{H}=3 / 4$
Also there is a high pressure region along the left side above the cylindrical base increased to the centre and upper left, which corresponds to where the flow enters the ventilator due to the momentum imparted on the rotor from the outside air.

The high pressure on the inside of the ventilator blades, discussed above, apparently makes a way into some distance through the ventilator in the mean flow, which would be the reason for the high pressure region above the cylindrical base. There is a low pressure area located in the region below the centre of cylindrical base which tends to left side, Fig. $8 \mathrm{a}$ and b. It is then attached to a low pressure area along the lower edge of the rotor, where the internal flow is confined by geometry of the rotating blades.

At $v_{f}=20 \mathrm{~m} \mathrm{sec}^{-1}$ at this cutting plane, it is once again difficult to define the structure in the pressure contours from experiment, Fig. 10c. There does appear to be at least one similarity with the low speed case, as a low pressure region can be seen close to the rotor on the left side of the wake. The streamtraces are once again similar in both cases, with less straightening at the high speed and the front pressure contours are almost the same for both speeds.

By checking the simulation outcomes at $\mathrm{v}_{\mathrm{f}}=20 \mathrm{~m} \mathrm{sec}^{-1}$, (Fig. 10a and b), it is apparent that the wake predicted by both turbulence models is still the same shape as the lower speed and the small left side low pressure region in the wake is once again as a result of flow separation at the trailing edge of the blade. Alike the results at lower free stream velocity, (Fig. 8), the wake is divided into two streams with the right one being larger than the left one however, the pressure contours in the wake appear closer together at $\mathrm{v}_{\mathrm{f}}=20 \mathrm{~m} \mathrm{sec}^{-1}$. The front high pressure regions, due to incidence of flow with the blades, exist still and continued some distance into the inside of the ventilator. The pressure distribution and the flow path through the ventilator blades are also similar to previous results at lower speed.

As it is illustrated yet, it can be concluded that, the airflow starts to split into two streams after passing the bottom of the rotating top. Meanwhile the airflow tends to proceed upward to the wake behind the rotating blades and join the swirl flow there. This can be due to the pressure difference and the effects of ventilator rotation. As it is shown in Fig. 8 and 10, the airflow is completely split into two streams after passing the rotating blades. The airflow which has passed the left side of the rotating blades comes to the low pressure region behind the blades and joins the airflow coming out through the blades. These two streams come 

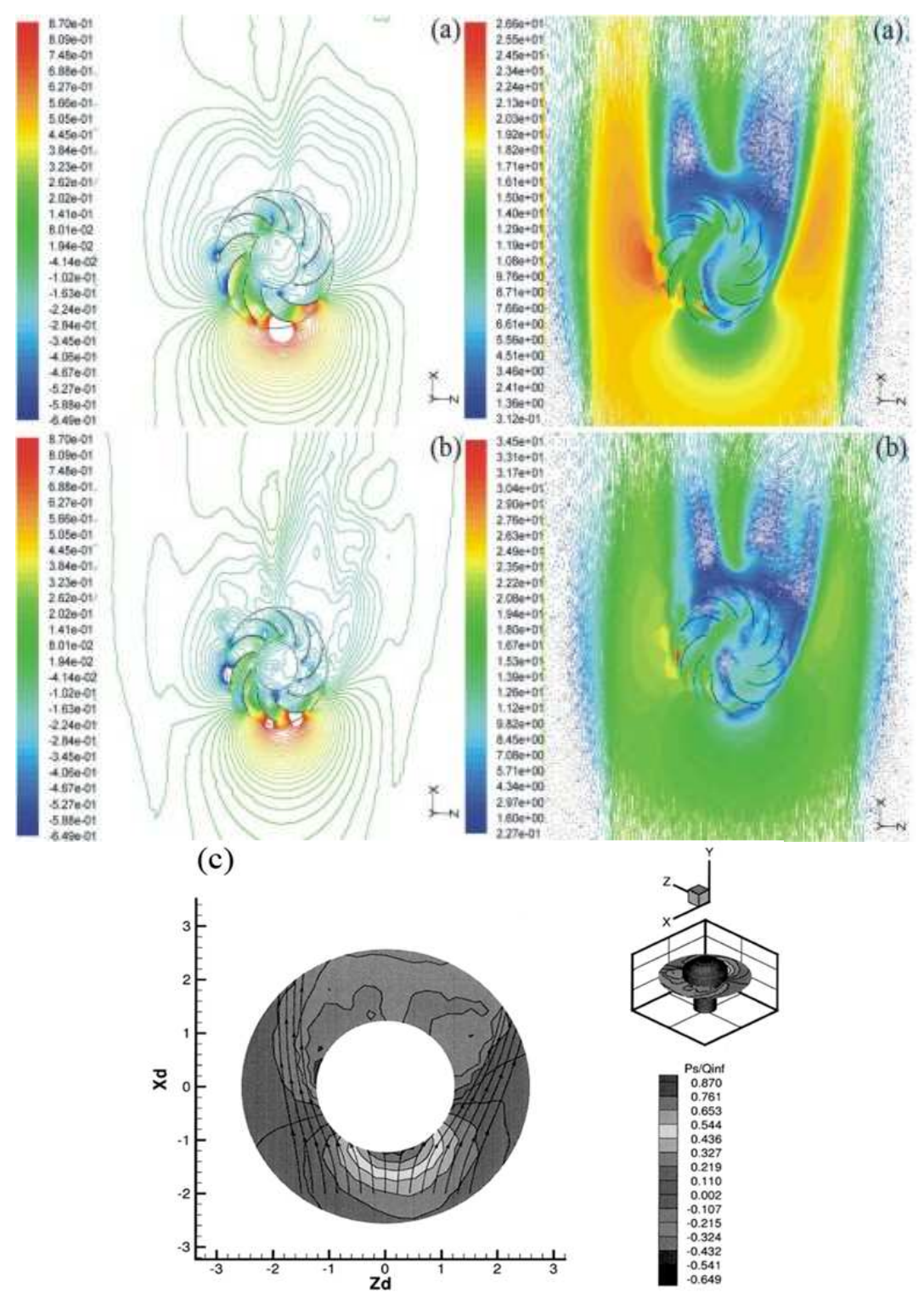

Fig. 10: Pressure contours and velocity magnitudes at $Y_{d}=1.015$ and $v_{f}=20 \mathrm{~m} \mathrm{sec}^{-1}$ for Realizable k- $\varepsilon$ (a), RSM (b) and experimental result (c), respectively

together with the stream coming from the bottom of the rotating top and form a gentle turbulent flow in this region. The airflow coming from the upper side, over the dome, helps the mentioned turbulent flow to proceed forward which creates the left stream. Similarly, the right stream is a combination of four different streams.

These results are in exact agreement with the flow visualization study by (Lai, 2003), which shows the airflow pattern around a turbine ventilator.
In conclusion, the entire flow field of the previously uninvestigated rotating turbine ventilator has been studied. It is demonstrated that the flow field around turbine ventilator is fully 3 -dimensional as the flow and pressure fields change conspicuously with the height of the cut-planes. When the flow is nondimensionalized, there appears to be a good agreement in velocity magnitude and pressure coefficient between the 2 test speeds of $v_{f}=7$ and $v_{f}=20 \mathrm{~m} \mathrm{sec}^{-1}$, which could indicate that the flow dynamics do not change noticeably. 
Am. J. Engg. \& Applied Sci., 3 (1): 159-170, 2010

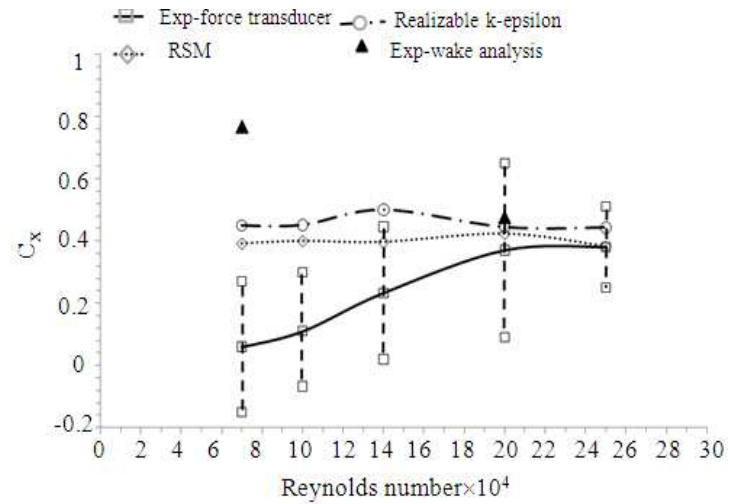

Fig. 11: Comparison of computed drag coefficient with experimental data

\section{DISCUSSION}

Aerodynamic forces results and discussions: The discussion of results on aerodynamic characteristics of a rotating turbine ventilator for different free stream velocities of $7,10,14,20$ and $25 \mathrm{~m} \mathrm{sec}^{-1}$ and comparison of simulation results to force transducer and wake analysis data from experiment (Pisasale, 2004) are given in this section. It must be noted that, in experiment force measurements were taken directly using an 'ATI Gamma' force transducer for entire range of velocities and compared to data from wake analysis. The results from wake analysis are given just for two velocities of 7 and $10 \mathrm{~m} \mathrm{sec}^{-1}$ which correspond to Reynolds number of $6.8 \times 10^{4}$ and $1.9 \times 10^{5}$.

The simulation results for coefficients of force in $\mathrm{x}$-direction, $\mathrm{C}_{\mathrm{x}}$, are shown in Fig. 11. As this component of force is parallel to flow direction, it is called drag coefficient. By checking the results, it is noticed that there is a tendency towards the experimental results when the free stream velocity is increasing.

The results shown from the wake analysis and the force transducer experiments, (Fig. 11), demonstrate a tendency for the stream to meet each other, when the free stream velocity is increasing. At lower speeds there are remarkable differences between the results from wake analysis and force transducer experiments. Significant differences are also exhibited between experiments and computations at same speeds. This is due to the miss-representation of the results from both experiments.

As it is illustrated in Fig. 11, at $v_{\mathrm{f}}=7 \mathrm{~m} \mathrm{sec}^{-1}$, the result from wake analysis seems to be over-estimated and this is due to the difficulty in resolving velocity in the wake. It must be mentioned that, Reynolds stresses also had been ignored in wake analysis experiment due

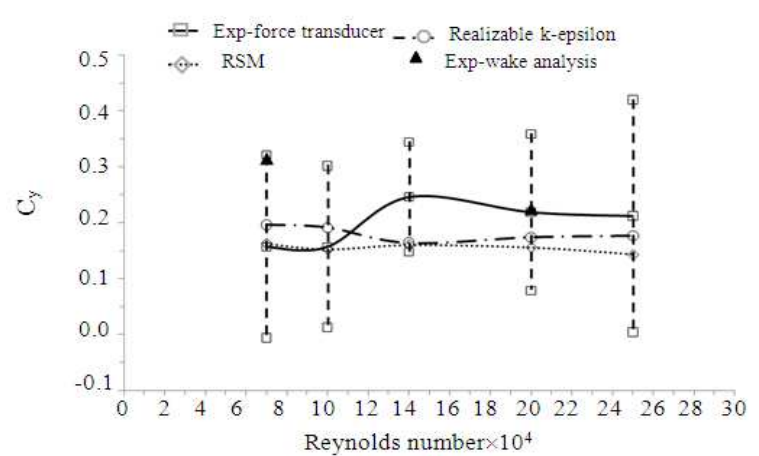

Fig. 12: Comparison of coefficient of force in $y$ direction with experimental data

to the difficulty in obtaining reliable data from the hotwire system. Despite being often expressed as viscous terms due to their relationship with strain, the Reynolds stresses actually come from the inertia term in NavierStokes equation. Consequently, when there is large turbulence and corresponding large Reynolds stresses, the force will be reduced.

On the other hand, by taking the geometry of turbine ventilator and direction of its rotation into account, it seems that the results from the force transducer experiment at low speeds especially at $\mathrm{v}_{\mathrm{f}}=7$ and $\mathrm{v}_{\mathrm{f}}=10 \mathrm{~m} \mathrm{sec}^{-1}$ are miss-represented by showing the negative amount for coefficient of drag. This means that the drag resultant forces are not of the same sense as the incident flow direction. These results obviously disagree with the physical situation.

As mentioned earlier, the change in angular velocity of the rotor with free stream magnitude is constant, which indicates flow similarity, supporting the flow visualization results, within the range tested. Therefore, it must be supported quantitatively, to some extent, by the proportions of force components. In contrary to the experiment, both turbulence models support the visualization results, quantitatively with a slight fluctuation.

At $v_{\mathrm{f}}=20 \mathrm{~m} \mathrm{sec}^{-1}$, the wake analysis result has decreased as it is expected due to resolving the difficulties about low velocities in the wake compare to results at $\mathrm{v}_{\mathrm{f}}=7 \mathrm{~m} \mathrm{sec}^{-1}$ and in contrary, the force transducer result once again has increased. At this speed the mentioned experimental results are in close agreement to each other. The results from both turbulence models are in good agreement with experiments.

By checking the results for coefficient of force in $y$ direction, Fig. 12, it is noticed that both computation results are in good agreement with experiments in entire range of velocities. 


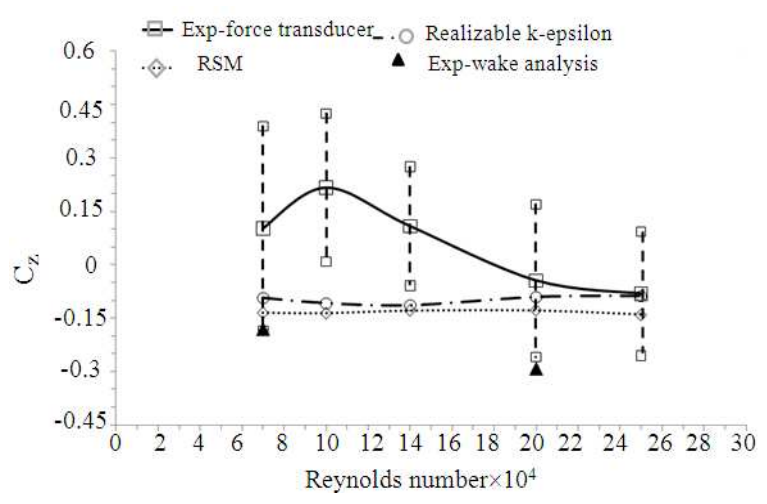

Fig. 13: Comparison of computed lift coefficient with experimental data

The results of computations in this direction as it is illustrated in Fig. 12 shows that, both turbulence models exhibit a constant trend in entire range of velocity and supports the flow visualization outcome once again.

Since the ventilator is rotating about the y-axis on the xz-plane, the lift force has been taken to act in the $\mathrm{z}$ direction and the lift coefficient is given by $\mathrm{C}_{\mathrm{z}}$ and is shown in Fig. 13. By checking the results on Fig. 13, once again it seems necessary to investigate the validation of experiments given for lift coefficient duo to the noticeable difference illustrated between the two sets of experiments.

In Fig. 13, the force calculated from wake analysis is generally less than that of the force from the transducer. Given that the non-dimensional flow is similar at both speeds of $v_{f}=7$ and $v_{f}=20 \mathrm{~m} \mathrm{sec}^{-1}$, which discussed earlier, this coefficient of force should be approximately constant. The flow visualization analysis indicates that the circulation varies along the rotor length and it seems the circulation induced by the rotor is likely to be less than approximated by the tangential velocity of the rotor. Consequently, the force coefficient should be less. Given all these considerations, it seems that the force coefficient measured by the transducer slightly miss-represented.

The wake analysis given here neglects the change in momentum and pressure of the internal flow and this cannot go unmentioned. The forces due to the internal dynamics should not have a large impact due to the comparatively smaller internal velocity compared to free stream velocity and the smaller volume of air that travels inside. As it is demonstrated by the flow visualization study, due to the momentum exchange on the rotor blades which give the rotor its torque, the high pressure inside the ventilator blades is in the negative $\mathrm{z}$ direction. Thus the resultant force inside the ventilator must be in z-direction and this consequently shows that the lift coefficient results from wake analysis are underpredicted and must be higher especially at high speeds.

As shown in Fig. 13, the results from both models show the same trend without any significant fluctuation in entire range of velocities and are in close agreement with each other. However, the results from force transducer experiment show a remarkable fluctuation and do not support the flow visualization results discussed earlier. At $\mathrm{v}_{\mathrm{f}}=20 \mathrm{~m} \mathrm{sec}^{-1}$, the results from force transducer and wake analysis seem to be more accurate than the results given at $v_{\mathrm{f}}=7 \mathrm{~m} \mathrm{sec}^{-1}$, however the wake analysis result is seem to be slightly under-predicted due to the neglecting of the flow inside the turbine ventilator.

\section{CONCLUSION}

In conclusion, the sequential course of simulation with a precise consideration of important parameters engaged in the action of rotating turbine ventilator, revealed the applicability of moving mesh method in modeling a turbine ventilator with a stationary base and rotating top. Also, the performance of two turbulence models was studied to predict the pressure distribution and velocity magnitude around and through the rotating turbine ventilator and the effect of circulation on the flow field. The Realizable $k-\varepsilon$ model showed a reasonable performance, however not as competence as the RSM model, but of much less computation time. The results from RSM model showed the best agreement to the experiment as expected due to ability of this model in providing a physically realistic and accurate prediction of the flow-field.

The results in this study present probably the very first flow-field investigation on the rotating turbine ventilator which demonstrated the continuity of flow through and around the rotating turbine ventilator. The visualization results indicate that the flow is fully threedimensional and the pressure distributions and velocity magnitudes are similar in two cases tested at two free stream velocities. This is also supported quantitatively, by the proportions of force components.

The superior understanding gained from this study would therefore, provide a foundation for future research into ventilator operation, which will result in the design of more efficient ventilators to improve the ventilation and consequently the human comfort.

\section{ACKNOWLEDGEMENT}

The researcher wish to thank Dr. Nor Mariah bt Adam for her close guidance, inspiration and invaluable help throughout this study. Also, I would like to thank 
Dr. Mohd Khairol Anuar Bin Mohd Ariffin for his kindness, information and suggestions during the project research.

\section{REFERENCES}

Cochran, B.C., 2004. A three-tiered approach for designing and evaluating performance characteristics of novel WECS. Proceeding of the 42nd AIAA Aerospace Sciences Meeting and Exhibit, Jan. 5-8, AIAA., Reno, Nevada, pp: 1362.

Dale, J.D. and M.Y. Ackerman, 1993. Evaluation of the performance of attic turbine ventilators. ASHRAE Trans., 99: 14-22.

Gatto, A., K.P. Byme, N.A. Ahmed and R.D. Archer, 2001. Mean and fluctuating pressure measurements over a circular cylinder in cross flow using plastic tubing. Exp. Fluids, 30: 43-46. DOI: $10.1007 / \mathrm{s} 003480000133$

Havens, P., 2004. A combined fan and wind turbine model for attic turbine ventilators. Energy Efficient and Healthy Buildings, ARE 318-E.

Khan, N., Y. Su and S.B. Riffat, 2008. A review on wind driven ventilation techniques. Energy Build., 40: 1586-1604. DOI: 10.1016/j.enbuild.2008.02.015

Kubota, T., D.T.H. Chyee and S. Ahmad, 2009. The effects of night ventilation technique on indoor thermal environment for residential buildings in hot-humid climate of Malaysia. Energy Build., 41: 829-839. DOI: 10.1016/j.enbuild.2009.03.008

Lai, C., 2003. Experiments on the ventilation efficiency of turbine ventilators used for building and factory ventilation. Energy Build., 35: 927-932. DOI: 10.1016/S0378-7788(03)00024-0

Lai, C., 2006. Prototype development of the rooftop turbine ventilator powered by hybrid wind and photo-voltaic energy. Energy Build., 38: 174-180. DOI: $10.1016 /$ j.enbuild.2005.06.004
Lai, C.M. and S. Kuo, 2005. Assessment of the potential of roof turbine ventilators for bathroom ventilation. Building Services Engineers: Technical note 26.2, pp: 173-179. DOI: $10.1191 / 0143624405 \mathrm{bt} 123 \mathrm{tn}$

Lakshminarayana, B., 1996. Fluid Dynamics and Heat Transfer of Turbomachinery. 1st Edn., John Wiley and Sons, Inc., ISBN: 0-47185546-4, pp: 809.

Marchman, J.F., 1982. Delta wing ventilator for improved passive ventilation. Proceeding of the 17th Intersociety Energy Conversion Engineering Conference, (IECEC'82), Los Angeles, USA., pp: $2-13$.

Meadows, V.H., 1929. US Patent 1,857,762, Rotary ventilator.

Pisasale, A.J., 2004. Novel aerodynamic techniques applied to the study of a rotating turbine ventilator. Doctoral Dissertation, The University of New South Wales, AAT 0807048, Sydney, Australia.

Rashid, D.M.H. and N.A. Ahmed, 2003. Study of Aerodynamic forces on a rotating wind driven ventilator. J. Wind Eng., 27: 63-72. DOI: 10.1260/030952403321833770

Reference Manual, 2006. FLUENT 6.3 User's Guide.

Revel, A., 1998. Testing of 2 wind driven roof ventilators. http://www.edmonds.com.au

West, S., 2001. Improving the sustainable development of building stock by the implementation of energy efficient, climate control technologies. Build. Environ., 36: 281-289. DOI: 10.1016/S03601323(00)00007-X

Wilson, D., 1994. Dispersion of exhaust gases from roof level stacks and vents on a laboratory building. Atmosph. Environ., 28: 3099-3111.DOI: 10.1016/1352-2310(94)E0067-T 